\title{
Harmonics detection using prony frequency analysis algorithm in transformer inrush current generation after fault clearance
}

\author{
Sandeep Wylie ${ }^{1}$, Pratibha Tiwari ${ }^{2}$ \\ M.Tech Student, Department of Electrical and Electronic Engineering (SSET), SHIATS, Allahabad India ${ }^{1}$ \\ Assistant Professor, Department of Electrical and Electronic Engineering (SSET), SHIATS, Allahabad India ${ }^{2}$
}

\begin{abstract}
The traditional infrastructure of power system industry is presently going through large amount of change that consensus over the improvement of power quality for fulfilling this requirement electronically automated devices having high sensitivity toward the disturbance in the power system signals are under the major consensus of research activities. The low power quality signal is responsible for malfunctioning of equipments that result in reduction of production and rise in the cost of production. In the modern age electronic convertors, facts and other thyristor controlled devices is combine with the new power transmission schemes. That produces harmonics current of high frequency order and brings large amount of current and voltage quality distortion. In this paper we are investigating and implementing the Prony harmonics analysis algorithm for estimation of harmonics in the voltage cycle available during saturable core transformer energizing the result are validate and compared with the short time Fourier analysis our proposed approach found to be more accurate and aliquant for non linear discrete data signals.
\end{abstract}

Keywords: Prony filter, THD, Short Time Fourier analysis, Saturable Core Transformer, Matlab.

\section{INTRODUCTION}

Many of the loads installed in present-day power systems networks. An increased effort by utilities and industries are harmonic current generators. Combined with the alike to discover improved mitigation methods that can impedance of the electrical system, the loads also produce keep harmonic distortion within allowable limits is harmonic voltages. The nonlinear loads may be viewed as foreseen. This section is devoted to describing three types both harmonic current generators and harmonic voltage of devices that leads to harmonics distortions, in no generators. Until 1970s, speed control of AC motors was primarily achieved using belts and pulleys [9].

Now, adjustable speed drives (ASDs) perform speed control functions very efficiently. ASDs are generators of large harmonic currents. Fluorescent lights uses less electrical energy for the same light output as incandescent lighting but produce substantial harmonic currents in the process. Due to increase of personal computer use it has resulted in harmonic current in commercial buildings. Harmonic distortion is no longer a phenomenon confined to industrial equipment and processes, where the first power quality concerns developed. Uninterruptible power supplies (UPSs), personal computers (PCs), and electronic and entertaining devices proliferate nowadays in commercial and residential installations. These special kinds of loads represent formidable sources of harmonic currents and they increase with the expanding use of video recorders, digital clocks, and other sensitive electronic equipment.

\section{HARMONIC SOURCE INVESTIGATION} particular order, a few of the more common nonlinear loads that surround us in our everyday life.Feilat (2006) presents an efficient method for the detection of the instantaneous flicker level. The technique was based on extracting the magnitudes, frequencies, and phase angles of all frequency components of the voltage envelope using Prony analysis. By reconstructing the voltage waveform as linear combination of sinusoids, Hilbert transform can be applied to the predicted signal to develop the envelope of the voltage waveform. Two cases of flicker with single low frequency and wide band frequency interharmonics are investigated using simulated voltage signals [3].

Costa et al.(2007)proposes a technique for harmonic analysis in electrical power systems. At the end, a frequency estimator, the Prony's method, has been matched to a Kalman filter. In the proposed technique, the sinusoid amplitudes of electrical power signals are estimated by the Kalman filter. The Kalman filter regressors are built up using the frequencies estimated by the Prony's method. The technique have been tested to both synthetically and experimental signals [4].

The interaction between power utility AC sources and Zygarlicki et al. (2009) presents a new modification of the customer loads will be further affected by distributed least-squares Prony's method for power-quality analysis in resources (often known as distributed generators or DGs) terms of estimation of harmonics and interharmonics in an that employ electronic switching technologies, electric power signal. The so-called reduced Prony's increasingly used by utilities and industry to better cope method can be competitive, in some specific case, to the with peak demand. The expanding presence of this type of Fourier transformation method and the classical LS DGs in distribution networks will contribute to an overall Prony's method. The modification constitutes in a specific rise in harmonic distortion in electric distribution

selection of a constant frequency vector in a Fourier-like 
manner leading to a remarkable reduction of the quality. Normally, Fourier transform(F.T) based computational burden. In addition, a sampling frequency approaches are used for supervising power system and an analysis window length can be selected to provide the numerical stability of the new algorithm [5].

\section{CONVENTIONAL METHODS OF HARMONIC DETECTION}

Many methods have been proposed for harmonic estimation in a power system. Most of them deal with this estimation as a totally nonlinear problem. Consequently, these methods either converge slowly, like GA algorithm or need accurate parameter adjustment to track dynamic and abrupt changes of harmonics amplitudes, like adaptive Kalman filter (KF). A novel hybrid approach, based on the decomposition of the problem into a linear and a nonlinear problem, was proposed by Joorabian et al. (2009), a linear estimator, i.e., Least Squares (LS), which is simple, fast and does not need any parameter tuning to follow harmonics amplitude changes, is used for amplitude estimation and an adaptive linear combiner called 'Adaline', which was very fast and very simple and was used to estimate phases of harmonics.

An improvement in convergence and processing time is achieved using this algorithm[6].The one-dimension frequency analysis based on DFT (Discrete FT ) is sufficient in many cases in detecting power disturbances and evaluating power quality (PQ). The character of the signal, using time-frequency analyses are performed by Szmajda et al.(2010). The most common known timefrequency representations (TFR) are spectrogram (SPEC) and Gabor Transform (GT). However, the method has a relatively low time-frequency resolution. The other TFR: Discreet Dyadic Wavelet Transform (DDWT), Smoothed Pseudo Wigner-Ville Distribution (SPWVD) and new Gabor-Wigner Transform (GWT) are described. The main feature of the transforms, on the basis of testing signals, was presented [7].

Zygarlicki et al. (2010) presents a new modification of the least-squares Prony's method for power-quality analysis in terms of estimation of harmonics and interharmonics in an electric power signal. The so-called reduced Prony's method can be competitive, in some specific case, to the Fourier transformation (F.T) method and the classical LS Prony's method. The modification constitutes in a specific selection of a constant frequency vector in a Fourier-like manner leading to a remarkable reduction of the computational burden. In addition, a sampling frequency and an analysis window length can be selected to provide the numerical stability of the new algorithm [8].

The origins of harmonics pollution can exist anywhere in the power system: on the generation side, load side, and even in the transmission network itself under modern electrical power system structures; as a result, identifying the harmonics will be a hard challenge facing the researchers [1].

In today's power systems, the proliferation of nonlinear loads has increased harmonic pollution. Harmonics cause many problems in connected power systems, such as reactive burden of power and low systems efficiency. Furthermore, harmonic selective active filters can be connected in power distribution systems to improve power harmonics. However, the accuracy of Fourier transform (F.T) is affected when these transient or time varying harmonics exist. Without prior knowledge of frequency components, some of the harmonics filters require PLL (Phase Locked Loops) or frequency estimators for identifying the specific harmonic frequency before the corresponding reference is generated.

\section{PRONY BASED PROPOSED METHODOLOGY}

For precision short time analysis of power waveforms fluctuations, the Prony's method can be used for the following reasons. The Prony analysis (P.A) is a parametric method of large complexity. This method outperformed its Fourier transform (F.T) counterpart in the accuracy of signal modelling and analysis for many practical signal processing situations.

The advantages of LS Prony's method includes precise estimation of frequency, amplitudes and phases, in addition to the ability to compute damping coefficients for the signal components, which can be used for transient analysis.

Since Prony analysis was first introduced into power system applications in 1990, it has been widely used for power system transient studies [2], but rarely used for power quality studies. Prony analysis is a method of fitting a linear combination of exponential terms to a signal as shown in Eqn.(1) [10]. Each term in Eqn.(1) has four elements: the magnitude $A_{n}$, the damping factor $\mathrm{n}$, the frequency $\mathrm{fn}$, and the phase angle n. Each exponential component with a different frequency is viewed as a unique mode of the original signal $y(t)$. The four elements of each mode can be identified from the state space representation of an equally sampled data record. The time interval between each sample is $\mathrm{T}$.

$y(t)=\sum_{n=1}^{N} A_{n} e^{\sigma_{n} t} \cos \left(2 \pi f_{n} t+\theta_{n}\right)=1,2,3, . N$

Using Euler's theorem and letting $\mathrm{t}=\mathrm{MT}$, the samples of $y(t)$ are rewritten as 1

$$
\begin{aligned}
& y_{M}=\sum_{n=1}^{N} B_{n} \lambda_{n}^{M} \\
& B_{n}=\frac{A_{n}}{2} e^{j \theta_{n}} \\
& \lambda_{n}=e^{\left(\sigma_{n}+j 2 \pi f_{n}\right) T}
\end{aligned}
$$

Prony analysis consists of three steps. In the first step, the coefficients of a linear predication model are calculated. The linear predication model (LPM) of order N, shown in Eqn.(1.4), is built to fit the equally sampled data record $y(t)$ with length $M$. Normally, the length $M$ should be at least three times larger than the order $\mathrm{N}$. 
$y_{M}=a_{1} y_{M-1}+a_{2} y_{M-2}+\cdots+a_{n} y_{M-N}$

Estimation of the LPM coefficients $\mathrm{n}$ a is crucial for the derivation of the frequency, damping, magnitude, and phase angle of a signal. In order to estimate these coefficients accurately, a matrix representation of the signal at various sample times can be formed by sequentially writing the linear prediction of $M y$ repetitively. By inverting the matrix representation, the linear coefficients $\mathrm{n}$ a can be derived from Eqn.(1.5). An algorithm, which uses singular value decomposition for the matrix inversion to derive the LPM coefficients, is called SVD algorithm.

$\left[\begin{array}{c}y_{N} \\ y_{N=1} \\ \vdots \\ y_{M-1}\end{array}\right]=\left[\begin{array}{cccc}y_{N=1} & y_{N=2} & \ldots & y_{0} \\ y_{N} & y_{N-1} & \ldots & y_{1} \\ \vdots & \vdots & & \vdots \\ y_{M-2} & y_{M-3} & \ldots & y_{M-N-1}\end{array}\right]\left[\begin{array}{c}a_{1} \\ a_{2} \\ \vdots \\ a_{n}\end{array}\right]$

In the second step, the roots $\lambda_{n}$ of the characteristic polynomial associated with the LPM from the first step are derived. The damping factor $\sigma_{n}$ and frequency fn are calculated from the root $\lambda_{n}$ according to Eqn.(1.6).

$$
\begin{aligned}
\lambda^{N}-a_{1} \lambda^{N-1}-\cdots-a_{N-1} \lambda-a_{N} \\
=\left(\lambda-\lambda_{1}\right)\left(\lambda-\lambda_{2}\right) \ldots\left(\lambda-\lambda_{n}\right) \ldots
\end{aligned}
$$

In the last step, the magnitudes and the phase angles of the signal are solved in the least square sense. According to Eqn.(1.2), Eqn.(1.3) is built using the solved roots $\lambda n$.

$$
\begin{aligned}
& \boldsymbol{Y}=\varphi B \\
& \boldsymbol{Y}=\left[\begin{array}{lll}
y_{0} & y_{1} & y_{M-1}
\end{array}\right]^{T}
\end{aligned}
$$

$\boldsymbol{\varphi}=\left[\begin{array}{cccc}1 & 1 & \ldots . . & 1 \\ \lambda_{1} & \lambda_{2} & \cdots . & \lambda_{N} \\ \vdots & \vdots & & \vdots \\ \lambda^{M-1} & \lambda^{M-1} & \ldots . . & \lambda_{N}^{M-1}\end{array}\right]$

$\boldsymbol{B}=\left[\begin{array}{llll}B_{1} & B_{2} & B_{3} & B_{3}\end{array}\right]$

The magnitude $A_{n}$ and phase angle $\theta_{n}$ are thus calculated from the variables $B_{n}$ according to (1.2). The greatest advantage of Prony analysis is its ability to identify the damping factor of each mode in the signal. Due to this advantage, transient harmonics can be identified accurately.

\section{SIMULINK MODELOF SATURABLE CORE TRANSFORMER CONECTED TO 3PHASE SOURCE}

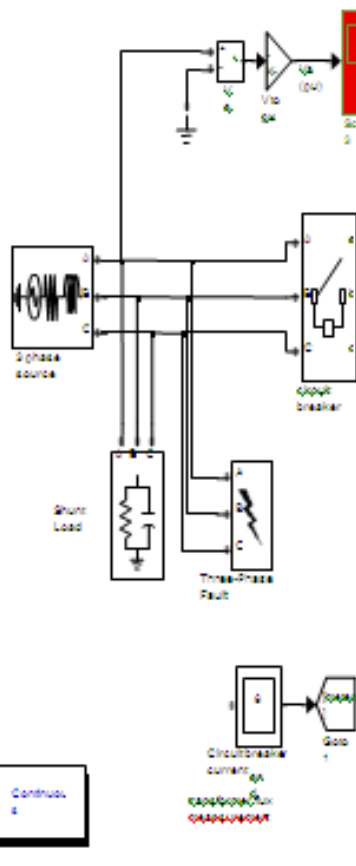

Figure 1

\section{SIMULINK RESULT AND DISCUSSION}

In saturable core Transformer, during the rise in the current the developed flux does not increseses proportionally but it saturate over the particular limit. Due to this, however, current is sinusoidal but the developed flux is non-sinusoidal i.e. it consist harmonics. This developed flux induced a voltage in the Transformer winding that voltage also consists of harmonics.

Whenever, a Transformer is connected to the source by a circuit braker the Transformer winding get energized suddenly and a large amount of current is induced due to sudden start of Transformer. This current is called 'Inrush current'. Since, this current is developing of flux and this flux will consist of saturation futures so this will cause generation of harmonics in the Transformer voltage. After some instant, the inrush current is decayed out hence, these harmonics are also get minimized.

As shown in figure, we can observe the 3 phase inrush current in the Transformer after closing of c.b. initially-

$I_{A}=2.8 \mathrm{amp}$. (Yellow line)

$I_{B}=-1.6 \mathrm{amp}$. (Blue line)

$I_{C}=-0.5 \mathrm{amp}$ (Green line)

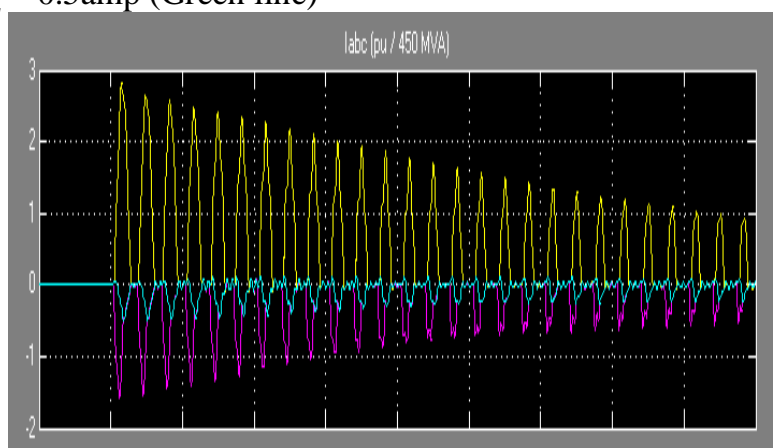

Figure 1(a): Transformer inrush current during energizing. 
Hence, the phase A has an inormushly high inrush current during the transformer energizing (See in fig. 1(a).

The voltage is measured for phase A because it has highest inrush current. Figure 4.2 shows the voltage in $\mathrm{Pu}-$

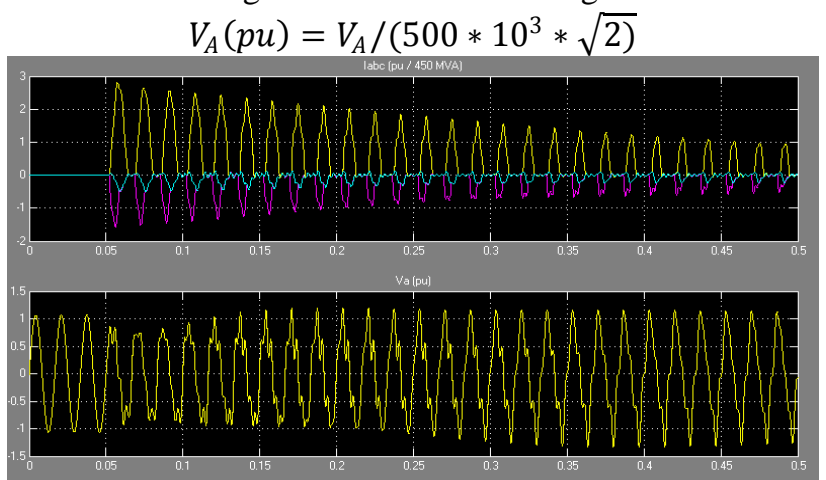

Figure 1(b): Inrush current Ia, Ib, Ic amp. (top) generation at 3 cycle and its effect on voltage $\mathrm{Va}$ pu (bottom).

In figure 1(b), we can see that upto the 3rd cycle i.e. $0.05 \mathrm{sec}$. the voltage is purely sinusoidal but after $3 \mathrm{rd}$ cycle as inrush current introduced we can observed that harmonics are introduced in the $\mathrm{V}_{\mathrm{A}}$. We can observed that the inrush current $\mathrm{I}_{\mathrm{A}}$ (yellow line) in fig. 4.2(top) is getting decayed gradually and it become almost $1 / 3$ rd after 0.5 sec. and parallel the harmonic distortion in our voltage is also varying and these distortion are also getting reduced as time reaches to $0.5 \mathrm{sec}$.

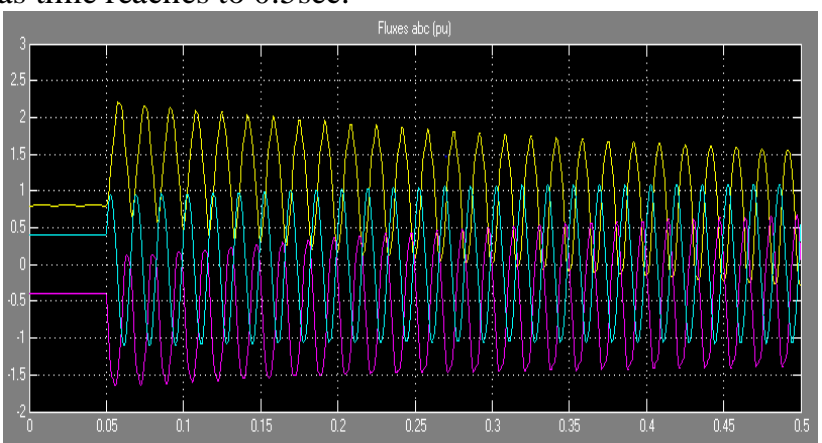

Figure 1 (c): Flux generated in transformer windings at primary side (yellow - pa.A, blue - ph. B and green -ph. C).

We have also observed the developed flux in our phase a winding in secondary side. This flux is obtained as-

$$
\begin{gathered}
V_{a}=\frac{d \emptyset}{d t} \\
\emptyset=\int V a d t
\end{gathered}
$$

Hence, by inserting integrator box i.e. (1/s) in the path of voltage $V_{a 2}$ we can observed the flux $\emptyset_{a}(\mathrm{Pu})$ at the transformer secondary side.We have used a multimeter base measurement for observing the current passing through the c.b. named as $\left(I_{c . b .}\right)$ and transformer flux or primary side. Circuit breker as shown in figure 1(a) and the primary winding flux along with flux induced in the secondary side of phase a winding as shown in figure 1(c). We can observe that at $0.5 \mathrm{sec}$, a large flux is generated specially in the phase a winding of the primary side of magnitude about $2.3 \mathrm{pu}$. In that flux decayed to $1.5 \mathrm{pu}$ at $\mathrm{t}=0.5 \mathrm{sec}$. Same flux is also observed in secondary side winding a flux. Figure 1(a) shows the all the flux phase a, $\mathrm{b}, \mathrm{c}$ in yellow, blue and green line while figure $1(\mathrm{~d})$ shows only flux of phase a.

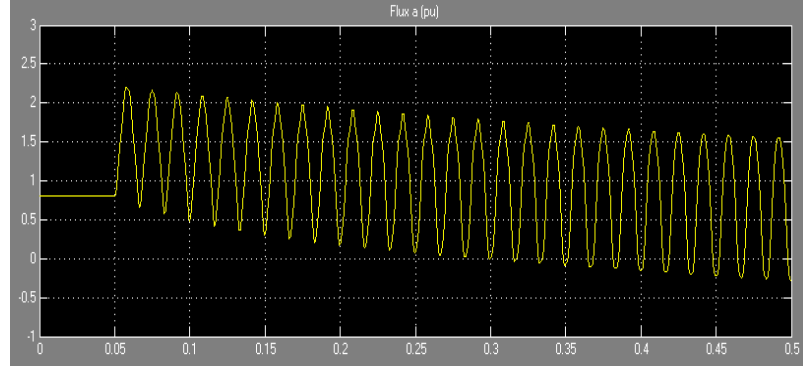

Figure $1(\mathrm{~d})$ : Flux generated in transformer windings of phase A at secondary side.

Table 1: Fourier Analysis results

\begin{tabular}{|l|l|}
\hline No. Of Cycles & THD \\
\hline 1 & $0.00 \%$ \\
\hline 2 & $0.00 \%$ \\
\hline 3 & $0.00 \%$ \\
\hline 4 & $27.48 \%$ \\
\hline 5 & $25.09 \%$ \\
\hline 6 & $25.69 \%$ \\
\hline 7 & $28.58 \%$ \\
\hline 8 & $31.08 \%$ \\
\hline 9 & $32.47 \%$ \\
\hline 10 & $32.65 \%$ \\
\hline 11 & $32.68 \%$ \\
\hline 12 & $31.65 \%$ \\
\hline 13 & $31.23 \%$ \\
\hline 14 & $30.79 \%$ \\
\hline 15 & $30.45 \%$ \\
\hline 16 & $30.19 \%$ \\
\hline 17 & $29.98 \%$ \\
\hline 18 & $29.79 \%$ \\
\hline 19 & $29.57 \%$ \\
\hline 20 & $29.29 \%$ \\
\hline & \\
\hline
\end{tabular}

Table 2: Prony Analysis results

\begin{tabular}{|l|l|}
\hline No. Of Cycles & THD \\
\hline 1 & $0.0001 \%$ \\
\hline 2 & $0.0001 \%$ \\
\hline 3 & $0.0001 \%$ \\
\hline 4 & $3.14 \%$ \\
\hline 5 & $14.93 \%$ \\
\hline 6 & $15 \%$ \\
\hline 7 & $17 \%$ \\
\hline 8 & $19 \%$ \\
\hline 9 & $18.64 \%$ \\
\hline 10 & $17 \%$ \\
\hline 11 & $14.7 \%$ \\
\hline 12 & $12 \%$ \\
\hline 13 & $9.3 \%$ \\
\hline 14 & $6.47 \%$ \\
\hline 15 & $4.5 \%$ \\
\hline 16 & $5 \%$ \\
\hline 17 & $7.5 \%$ \\
\hline 18 & $10.6 \%$ \\
\hline 19 & $13.6 \%$ \\
\hline 20 & $16 \%$ \\
\hline & \\
\hline
\end{tabular}


As we can see from the Table $1 \& 2$ we calculated Total Harmonic Distortion, but from Prony Analysis we can calculate the THD, Order, Magnitude, phase and the most important we calculate the damping factor of the signals that at which rate harmonic is going to decay, we also observed during analysis that the $4^{\text {th }}$ harmonics will sustained for long time with the fundamental component as compared to other harmonic.

\section{ACKNOWLEDGEMENT}

The author sincerely, thanks Dissertation guide SHIATS Allahabad, India to carried out this Research work

\section{CONCLUSION}

This paper shows that the harmonics content and its variation during the saturable core transformer energizing due to sudden state transition of the circuit breaker. We have developed a 3 phase source based power system simulink model using Matlab.In summary, it has been concluded as both Fourier and Prony confirms the presences of 4th harmonic as the most affective frequency constant that causes the distortion in voltage waveform. As the number of cycles increases the $\%$ magnitude of 4 th harmonic is found to be increases and after the 8th cycle the magnitude of 4th harmonic is suddenly decreases and it decays to the range of $10.5 \mathrm{v}$ up to the 13th cycle and after this cycle the th harmonic again start razing and present as a most affective harmonic content in each cycle. In this way out of 17 cycle in almost 9 cycles, 4th harmonic is present with largest magnitude. Thus conclusion is also verified by the fourier analysis results. Therefore our proposed approach is found to be better.

\section{REFERENCES}

[1] J. Arrillaga, MHJ Bollen, and NR Watson. Power quality following deregulation. Proceedings of the IEEE, 88(2):246-261, 2000.

[2] JF Hauer, CJ Demeure, LL Scharf, 1990 Initial results in Prony analysis of power system response signals. IEEE Transactions on Power Systems 5(1), 80-89.

[3] Feilat, E.A. 2006. Prony analysis technique for estimation of the mean curve of lightning impulses. IEEE Trans. Power Del., 21(4), 2088-2090.

[4] Costa, F.F., Cardoso , A.J.M., Fernandes, Darlan A. 2007. Harmonic Analysis Based on Kalman Filtering and Prony s Method. POWERENG 2007, Setubal, Portugal, 696-701.

[5] Zygarlicki, J., Zygarlicka, M., Mroczka, J. 2009. Prony's method in power quality analysis. Energy Spectrum, 4(2), 26-30.

6] Joorabian, M., Mortazavia, S.S.,Khayyami, A.A. 2009. Harmonic estimation in a power system using a novel hybrid Least SquaresAdaline algorithm. Electric Power Systems Research, 79(1),107-116.

[7] Szmajda, M., Górecki, K., Mroczka, J. 2010. Gabor transform, SPWVD, Gabor-Wigner transform and wavelet transform - tools for power quality monitoring. Metro. and Meas. Syst., 16(3), 383-396.

[8] Zygarlicki J., Zygarlicka M., Mroczka, J., Latawiec, K. 2010. A reduced Prony's method in power quality analysis - parameters selection. IEEE Transactions on Power Delivery, 25(2), 979- 986.

[9] Frank, J.M., 1997. Origin, development and design of K-factor transformers, IEEE Ind.Appl. Mag. , Sept/Oct.

[10] Hildebrand, F. B. 1956. Introduction to Numerical Analysis, McGraw-Hill Book Company,Inc.

[11] StandardEN5016 (2010)-Voltage characteristics of electricity supplied by public distribution networks.

[12] Standard IEC 61000-4-7 (2009) - Testing and measurement techniques - General guide on harmonics and interharmonics measurements and instrumentation, for power supply systems and equipment connected thereto.

\section{BIOGRAPHIES}

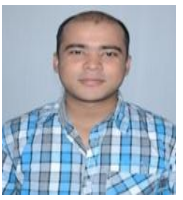

Sandeep Wylie belongs to Dehradun, Uttarakhand receive his Bachelor of technology from SHIATS University, Allahabad in 2012.He is pursuing M.Tech in Electrical Engg.(Power System)from SHIATS University, Allahabad UP-India.

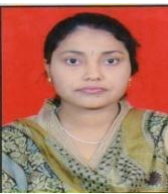

Er.Pratibha Tiwari, belong to Allahabad, Receive her Bachelor of technology from VPS Purvanchal University Jaunpur in 2002. She obtained her M.Tech in Electrical Eng., (Control and Instrumentation) from MNNII Allahabad in 2006.Presently she is working as a Asst. Prof in Dept. of SSET SHIATS Allahabad UP, India. 